East Asian Mathematical Journal

Vol. 29 (2013), No.3, pp. 255-258

http://dx.doi.org/10.7858/eamj.2013.016

\title{
EMBEDDING PROPERTIES IN NEAR-RINGS
}

\author{
YONG UK CHO
}

\begin{abstract}
In this paper, we initiate the study of zero symmetric and constant parts of near-rings, and then apply these to self map near-rings.

Next, we investigate that every near-ring can be embedded into some self map near-ring, and every zero symmetric near-ring can be embedded into some zero symmetric self map near-ring.
\end{abstract}

\section{Introduction}

Throughout this paper, a near-ring $R$ is an algebraic system $(R,+, \cdot)$ with two binary operations, say + and $\cdot \operatorname{such}$ that $(R,+)$ is a group (not necessarily abelian) with neutral element $0,(R, \cdot)$ is a semigroup and $a(b+c)=a b+a c$ for all $a, b, c$ in $R$. We note that obviously, $a 0=0$ and $a(-b)=-a b$ for all $a, b$ in $R$, but in general, $0 a \neq 0$ and $(-a) b \neq-a b$.

If $R$ has a unity 1 , then $R$ is called unitary. An element $d$ in $R$ is called distributive if $(a+b) d=a d+b d$ for all $a$ and $b$ in $R$.

We consider the following substructures of near-rings: Given a near-ring $R$, $R_{0}=\{a \in R \mid 0 a=0\}$ which is called the zero symmetric part of $R$,

$$
R_{c}=\{a \in R \mid 0 a=a\}=\{a \in R \mid r a=a, \text { for all } r \in R\}=\{0 a \mid a \in R\}
$$

which is called the constant part of $R$, and $R_{d}=\{a \in R \mid a$ is distributive $\}$ which is called the distributive part of $R$.

We note that $R_{0}$ and $R_{c}$ are subnear-rings of $R$, but $R_{d}$ is not a subnear-ring of $R$. A near-ring $R$ with the extra axiom $0 a=0$ for all $a \in R$, that is, $R=R_{0}$ is said to be zero symmetric, also, in case $R=R_{c}, R$ is called a constant near-ring, and in case $R=R_{d}, R$ is called a distributive near-ring.

Let $(G,+)$ be a group (not necessarily abelian). We may obtain some examples of near-rings as following:

In the set

$$
M(G)=\{f \mid f: G \longrightarrow G\}
$$

of all the self maps of $G$, if we define the sum $f+g$ of any two mappings $f, g$ in $M(G)$ by the rule $x(f+g)=x f+x g$ for all $x \in G$ and the product $f \circ g$ by

Received May 7, 2011; Revised September 25, 2011; Accepted February 5, 2013

2000 Mathematics Subject Classification. 16Y30.

Key words and phrases. zero symmetric part, constant part, self map near-ring and embedding. 
the rule $x(f \circ g)=(x f) g$ for all $x \in G$, then $(M(G),+, \circ)$ becomes a near-ring. It is called the self map near-ring on the group $G$. Also, we can define the substructures of $(M(G),+, \circ)$ as following: $M_{0}(G)=\{f \in M(G) \mid 0 f=0\}$ and $M_{c}(G)=\{f \in M(G) \mid f$ is constant $\}$.

For the remainder basic concepts and results on near-rings, we refer to [3].

\section{Some embedding Properties in near-rings}

Let $R$ and $S$ be two near-rings. Then a mapping $\theta$ from $R$ to $S$ is called a near-ring homomorphism if (i) $(a+b) \theta=a \theta+b \theta$, (ii) ( $a b) \theta=a \theta b \theta$. Obviously, $R \theta<S$ and $T \theta^{-1}<R$ for any $T<S$.

Let $R$ be any near-ring and $G$ an additive group. Then $G$ is called an $R$-group if there exists a near-ring homomorphism

$$
\theta:(R,+, \cdot) \longrightarrow(M(G),+, \cdot) .
$$

Such a homomorphism $\theta$ is called a representation of $R$ on $G$, we may write that $x r$ (right scalar multiplication in $R$ ) for $x(r \theta)$ for all $x \in G$ and $r \in R$. If $R$ is unitary, then $R$-group $G$ is called unitary. Thus a (unitary) $R$-group is an additive group $G$ satisfying (i) $x(a+b)=x a+x b$, (ii) $x(a b)=(x a) b$ and (iii) $x 1=x$ ( if $R$ has a unity 1 ), for all $x \in G$ and $a, b \in R$.

Evidently, every near-ring $R$ can be given the structure of an $R$-group (unitary, if $R$ is unitary) by right multiplication in $R$. Moreover, every group $G$ has a natural $M(G)$-group structure, from the representation of $M(G)$ on $G$ by applying the $f \in M(G)$ to the $x \in G$ as a scalar multiplication $x f$.

Proposition 2.1. Let $(G,+)$ be a group and let $\Phi$ a subset of endomorphisms of $(G,+)$ containing zero endomorphism $\zeta$. Then the set

$$
M_{\Phi}(G)=\{f \in M(G) \mid f \circ \phi=\phi \circ f, \forall \phi \in \Phi\}
$$

is a unitary zero symmetric subnear-ring of $M(G)$.

Proof. Let $f, g \in M_{\Phi}(G)$. Then $f \circ \phi=\phi \circ f$ and $g \circ \phi=\phi \circ g$ for any $\phi \in \Phi$, and so we have, since $\phi$ is an endomorphism,

$$
\begin{gathered}
x[(f-g) \circ \phi]=(x(f-g)) \phi=(x f-x g) \phi \\
=(x f) \phi-(x g) \phi=x(f \circ \phi)-x(g \circ \phi)=x(\phi \circ f)-x(\phi \circ g) \\
=(x \phi) f-(x \phi) g=(x \phi)(f-g)=x[\phi \circ(f-g)],
\end{gathered}
$$

for all $x \in G$. Hence $(f-g) \circ \phi=\phi \circ(f-g)$, and so $f-g \in M_{\Phi}(G)$. This implies that $\left(M_{\Phi}(G),+\right)$ is a subgroup of $(M(G),+)$.

Next, for any $f, g \in M_{\Phi}(G)$, we have that

$$
(f \circ g) \circ \phi=f \circ(g \circ \phi)=f \circ(\phi \circ g)=(\phi \circ f) \circ g=\phi \circ(f \circ g),
$$

for any $\phi \in \Phi$. Hence $f \circ g \in M_{\Phi}(G)$. Consequently, $\left(M_{\Phi}(G),+, \circ\right)$ is a subnear-ring of $(M(G),+, \circ)$.

Finally, let $f \in M_{\Phi}(G)$. Since $\zeta \in \Phi$, we see that $\zeta \circ f=f \circ \zeta=\zeta$. Therefore $M_{\Phi}(G)$ is zero symmetric with identity $1_{G}$. 
Corollary 2.2. For any group $(G,+),\left(M_{0}(G),+, \circ\right)$ is a zero symmetric subnear-ring of $(M(G),+, \circ)$. Moreover, $M_{0}(G)=M(G)_{0}$, where $M(G)_{0}$ is a zero symmetric part of $M(G)$.

Proof. The first paragraph is immediately from the Proposition 2.1.

Next, clearly, $M_{0}(G) \subseteq M(G)_{0}$, because every element of $M_{0}(G)$ is zero symmetric by Proposition 2.1.

Conversely, let $f \in M(G)_{0}$. Then $\zeta \circ f=\zeta$, that is, for any $x \in G$, $(x \zeta) f=x \zeta$. This implies that $0 f=0$. Hence, $f \in M_{0}(G)$.

Remark 1. Let $G$ be an additive group. Then we see that $M_{c}(G)=M(G)_{c}$, where $M(G)_{c}$ is the constant part of $M(G)$.

Indeed, if $f \in M_{c}(G)$, then $f$ is constant, say, $f=c$. From this,

$$
x(\zeta \circ f)=(x \zeta) f=0 f=c=x f,
$$

for all $x \in G$. Hence $\zeta \circ f=\zeta$, and so $f \in M(G)_{c}$.

Conversely, if $f \in M(G)_{c}$, then $\zeta \circ f=f$, that is, $0 f=(x \zeta) f=x f$, for all $x \in G$. Hence $f$ is constant, so that $f \in M_{c}(G)$.

Note that from Corollary 2.2 and Remark $1, M_{0}(G)$ is a zero symmetric near-ring and $M_{C}(G)$ is a constant near-ring.

Lemma 2.3. Let $f: R \longrightarrow S$ be a near-ring homomorphism. Then the following conditions are true. (1) $R_{0} f \subseteq S_{0}$. (2) $R_{c} f \subseteq S_{c}$.

Proof. (1) Let $y \in R_{0} f$ which is in $S$. Then there exists $a \in R_{0}$ such that $y=a f$, where $0 a=0$. Thus

$$
0 y=0(a f)=0 f a f=(0 a) f=0 f=0 .
$$

Hence $y \in S_{0}$.

(2) Let $y \in R_{c} f$ which is in $S$. Then there exists $a \in R_{c}$ such that $y=a f$, where $0 a=a$. Thus

$$
0 y=0(a f)=0 f a f=(0 a) f=a f=y .
$$

Hence $y \in S_{c}$.

Let $f: R \longrightarrow S$ be a near-ring monomorphism. We know that $R f$ is a subnear-ring of $S$, and so $f: R \longrightarrow R f$ is a near-ring isomorphism. Thus $S$ has an isomorphic copy of $R$ as a subnear-ring. We say that $R$ is embedded into $S$, and $f$ is an embedding.

Proposition 2.4. Let $(R,+, \cdot)$ be a near-ring and $(G,+)$ a group containing $(R,+)$ as a proper subgroup. Then $(M(G),+, \circ)$ is a unitary near-ring and has a subnear-ring isomorphic to $(R,+, \cdot)$. That is, every near-ring can be embedded into a near-ring with identity. 
Proof. For any $a \in R$, we may define a map $f_{a} \in M(G)$ by

$$
x f_{a}=a \text {, if } x \notin R \text {, and }=x a \text {, if } x \in R .
$$

Thus we may obtained the map $\Psi: R \longrightarrow M(G)$ which is defined by $a \Psi=$ $f_{a}$. We will proceed to show that $\Psi$ is a near-ring monomorphism. For any $a, b \in R$, since $(a+b) \Psi=f_{(a+b)}$ we have that

$$
x f_{(a+b)}=a+b \text {, if } x \notin R, \text { and }=x(a+b)=x a+x b, \text { if } x \in R .
$$

Also, we have that

$$
x\left(f_{a}+f_{b}\right)=x f_{a}+x f_{b}=a+b \text {, if } x \notin R, \text { and }=x a+x b \text {, if } x \in R .
$$

Thus

$$
(a+b) \Psi=f_{(a+b)}=f_{a}+f_{b}=a \Psi+b \Psi .
$$

On the other hand, from $(a b) \Psi=f_{(a b)}$ we have that

$$
x f_{(a b)}=a b, \text { if } x \notin R, \text { and }=x(a b)=(x a) b, \text { if } x \in R .
$$

Also, we have that

$$
x\left(f_{a} \circ f_{b}\right)=\left(x f_{a}\right) f_{b}=a b \text {, if } x \notin R \text {, and }=(x a) f_{b}=(x a) b \text {, if } x \in R .
$$

Thus

$$
(a b) \Psi=f_{(a b)}=f_{a} \circ f_{b}=a \Psi \circ b \Psi .
$$

Hence $\Psi$ is a near-ring homomorphism from $R$ into $M(G)$.

Next, it remains to show that $\Psi$ is injective. Let $a, b \in R$ with $a \neq b$. We want to show that $a \Psi=f_{a} \neq f_{b}=b \Psi$. For $x \notin R$, we know that $x f_{a}=a \neq b=x f_{b}$. Consequently, we obtain what we wanted.

Corollary 2.5. For any group $(G,+), R_{0} \Psi \subseteq M_{0}(G)$ and $R_{c} \Psi \subseteq M_{c}(G)=$ $M(G)_{c}$.

From Corollary 2.5, we obtain the following important result as in ring theory.

Proposition 2.6. If $R$ is any zero symmetric near-ring, then $R$ can be embedded into some zero symmetric near-ring with identity.

\section{References}

[1] Y. U. Cho Quotient substructures of R-groups, J Applied Mathematics and Informatics 28 No 1-2, (2010), 345-349.

[2] J.D.P. Meldrum Upper faithful D.G. near-rings, Proc. Edinburgh Math. Soc. 26, (1983), 361-370.

[3] G. Pilz Near-rings, , North Holland Publishing Company, Amsterdam, New York, Oxford 1983.

YONG UK CHO

Department of Mathematics, College of Education, Silla University, Pusan 617736, Korea,

E-mail address: yucho@silla.ac.kr 